\section{Atención de enfermería pediátrica en neurofibromatosis tipo 1. Caso clínico}

\author{
Pediatric nursing care in type 1 neurophibromatosis 1. \\ Clinical case
}

\author{
Cuidados de enfermagem pediátrica no tipo de \\ neurophibromatose 1. Caso clínico
}

\author{
Marlin Angélica Guía Yanes \\ mguia28@hotmail.com
}

Recibido mayo 2018 / Revisión junio 2018 / Aceptado 1 de agosto 2018

\section{RESUMEN}

Introducción: Se presenta un caso clínico de una escolar con diagnóstico de Neurofibromatosis tipo 1 (NF-1) enfermedad descrita en 1793 por von Tilesius y se caracteriza por ser una enfermedad progresiva multisistémica de herencia autosómica dominante. Objetivo: Analizar las intervenciones de enfermería en la NF-1 como patología de aparición poco frecuente. Materiales y Métodos: Posterior a la valoración de enfermería se procedió a determinar los diagnósticos y establecer el plan de acción con base a las necesidades detectadas, para ello se utilizó el Proceso de Atención que es la metodología del trabajo enfermero. Discusión: Las intervenciones de enfermería fueron dirigidas a las necesidades interferidas que presentó la escolar a fin de mejorar su condición y brindar educación oportuna a los padres. Conclusión: Es importante el diagnóstico precoz de esta patología, por ello tanto la enfermera clínica como la comunitaria, deben estar al tanto de los signos durante la valoración pediátrica, realizar las intervenciones acordes a la evolución de la patología y necesidades interferidas y remitir al especialista.

Palabras clave: neurofibromatosis 1; enfermería; atención de enfermería

\section{ABSTRACT}

Introduction: A clinical case of a schoolgirl with a diagnosis of neurofibromatosis type 1 (NF-1) disease described in 1793 by von Tilesius is presented and is characterized as a progressive multisystemic disease of autosomal dominant inheritance. Objective: To analyze the nursing interventions in the NF-1 as an infrequent pathology. Materials and Methods: After the nursing assessment, the diagnoses were determined and the action plan was established based on the needs detected, for this purpose the Care Process was used, which is the methodology of nursing work. Discussion: Nursing interventions were directed to the interfered needs presented by the school in order to improve their condition and provide timely education to parents. Conclusion: It is important the early diagnosis of this pathology, therefore both the clinical and community nurse, should be aware of the signs during the pediatric assessment, perform the interventions according to the evolution of the pathology and interfered needs and refer to the specialist.

Key words: neurofibromatosis 1; nursing; nursing care
MG: Universidad Central de Venezuela, Venezuela. 


\section{RESUMO}

MG: Universidad Central de Venezuela, Venezuela.

\begin{abstract}
Introdução: Apresenta-se um caso clínico de uma estudante com diagnóstico de neurofibromatose tipo 1 (NF-1), descrita em 1793 por von Tilesius, caracterizada como uma doença multissistêmica progressiva de herança autossômica dominante. Objetivo: Analisar as intervenções de enfermagem na NF-1 como uma patologia pouco frequente. Materiais e Métodos: Após a avaliação de enfermagem, os diagnósticos foram determinados e o plano de ação foi estabelecido com base nas necessidades detectadas, para esse fim foi utilizado o Processo de Cuidados, que é a metodologia do trabalho de enfermagem. Discussão: As intervenções de enfermagem foram direcionadas às necessidades interferentes apresentadas pela escola, a fim de melhorar sua condição e proporcionar educação oportuna aos pais. Conclusão: É importante o diagnóstico precoce dessa patologia, pois tanto o enfermeiro clínico quanto o comunitário devem estar atentos aos sinais durante a avaliação pediátrica, realizar as intervenções de acordo com a evolução da patologia e necessidades interferentes e encaminhar ao especialista. .
\end{abstract}

Palavras-chave: neurofibromatose 1; enfermagem; cuidados de enfermagem

\section{INTRODUCCIÓN}

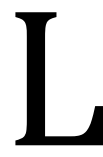
a Neurofibromatosis tipo I NF-1 fue descrita en 1793, por von Tilesius. La NF-1 es una enfermedad progresiva multisistémica de herencia autosómica dominante que tiene una expresividad muy variable. El propósito de este caso fue analizar las intervenciones de enfermería en laNF-1 como patología poco frecuente de aparición con una prevalencia de uno caso por cada 3.500 individuos. Es por ello que resulta interesante observar el patrón fisiopatológico y las complicaciones que se producen a raíz del avance de la patología, para determinar las intervenciones del personal de enfermería más acordes según las necesidades y limitaciones funcionales que presentan los niños e identificar los signos de evolución de esta.

La neurofibromatosis se ha descrito de varias maneras, principalmente como la neurofibromatosis tipo 1 (NF-1), denominada previamente o enfermedad de von Recklinghausen y la Neurofibromatosis tipo 2 (NF-2) (1).

En 1793, la NF-1 fue descrita por von Tilesius. En el año 1882, la primera descripción detallada de la NF1 corresponde al profesor alemán de patología de la Universidad de Estamburgo, Friedrich Daniel von Recklinghausen (1833-1910) (1), publicando en ese mismo año la obra titulada "Los fibromas múltiples de la piel y su relación con los neuromas múltiples" (2) en el que relacionaba los neurofibromas superficiales de la piel con los internos y proponía para la enfermedad, el nombre de "facomatosis", del griego fakos lunar o antojo o marca de nacimiento (3). La patología observada se caracterizaba precisamente por numerosas marcas de nacimiento o manchas de color café con leche. Es una enfermedad poco común con una prevalencia de uno de cada 3.500 individuos (2).

La NF-1 es una enfermedad progresiva multisistémica neurocutánea de herencia autosómica dominante, que tiene una expresividad muy variable y define un grupo de afecciones, caracterizada por el desarrollo de múltiples neurofibromas o schwannomas, con o sin múltiples manchas café con leche. Los neurofibromas son tumores benignos que provienen de los pequeños o grandes nervios; están compuestos principalmente 
por células de Schwann y fibroblastos. Estos tumores contienen además células perineurales, endoteliales y un pequeño número de células neurales (1).

El gen de la NF-1 consta de 62 exones, es localizada en el cromosoma 17, en la banda q11.2 y se origina en una mutación en la línea germinal de este gen codificante de la neurofibromina. La neurofibromina, es una proteína de $327 \mathrm{kDa}$, que activa a la GTPasa, la cual regula la actividad biológica de otras proteínas codificadas por protooncogenes de la familia Ras (4) y se expresa en las células de Schwann, los melanocitos, los leucocitos, la glándula suprarrenal y, entre otros tejidos, en el sistema nervioso central (5).

De manera biomolecular, la NF-1 pertenece al grupo de las rasopatías, una serie de trastornos genéticos derivados de mutaciones germinales en los genes implicados en la vía de señalización Ras/Mitogen activated protein kinase (MAPK), una cascada metabólica que se encarga de regular la proliferación y la diferenciación celular, la organogénesis, la plasticidad sináptica, el crecimiento, la apoptosis y el envejecimiento celular (5).

La NF-1 se caracteriza por la presencia de manchas de color llamadas "café con leche", nódulos de Lisch en el iris, pecas axilares 0 inguinales $y$ múltiples tumoraciones de tejido nervioso o neurofibromas que pueden estar ubicados en cualquier parte del cuerpo, ya sea en el tejido subcutáneo o en los nervios del cerebro (pares craneales) y de la médula espinal (nervios o pares raquídeos) $(6,7)$.

Es frecuente que los pacientes con NF1 presenten dificultades de aprendizaje, trastornos cardiacos, hipertensión, determinadas anomalías fenotípicas como macrocefalia, neuropatías, tumores de nervios periféricos, talla baja, trastorno neuroendocrino debido a tumores hipotalámicos, pubertad anormal, feocromocitoma, trastornos de la pigmentación e incluso, predisposición al cáncer gastrointestinal, pueden presentar sangrado u obstrucción debido a neurofibromas, estreñimiento (5).

Para el diagnóstico de neurofibromatosis, un paciente debe tener al menos 2 de los siguientes 7 criterios: 6 o más manchas "café con leche", iguales o mayores de $5 \mathrm{~mm}$ en pacientes prepúberes y de $15 \mathrm{~mm}$ de diámetro en pacientes postpúberes; 2 o más neurofibromas de cualquier tipo, o uno plexiforme, presencia de pecas en axilas o ingles, glioma del nervio óptico; 2 ó más nódulos de Lisch (harmatomas del iris pigmentaciones a nivel ocular translúcidas de coloración acastañada en el iris, conocidos como nódulos de Lisch) (4); lesión ósea definida como displasia del esfenoides o adelgazamiento de la cortical de los huesos largos con 0 sin pseudoartrosisyun familiar de primer grado de consanguineidad afectado (padre, hermano o hijo) de acuerdo con los criterios previos.

El diagnóstico diferencial se debe establecer con el síndrome de Legius (8), donde únicamente se presentan manchas de color café con leche y cursa con menor gravedad. Otros diagnósticos diferenciales a considerar son el síndrome de 34 Noonan con lentiginosis (síndrome LEOPARD) y el síndrome de McCune-Albright (8).

No hay cura para la NF-1, pero hay maneras de tratar algunos de sus efectos. Se pueden extirpar los tumores de la piel que causan dolor o desfiguración mediante cirugía. Sin embargo, con frecuencia vuelven a crecer. Los gliomas ópticos (tumores en el ojo) que afectan a la vista pueden tratarse con cirugía y/o radioterapia. La escoliosis puede tratarse mediante cirugía, aparatos ortopédicos, o ambos. Los tumores que causan dolor o pérdida de alguna función se pueden extirpar dependiendo de su localización. Si no hay complicaciones, la expectativa de 
vida de las personas con NF es casi normal.

Las complicaciones de la NF-1 se deben a afectación directa de los diversos sistemas por los neurofibromas, a un riesgo incrementado de malignización. La mayoría son fibrosarcomas, pero también otros tumores malignos, inclusive leucemias.

El profesional de enfermería fundamenta su trabajo en el "Proceso de Enfermería" que consiste en la aplicación del método científico a la práctica del cuidado. Para ello se apoya de las etapas de valoración, diagnóstico, planeación y evaluación, y a partir de allí, fija las pautas a seguir.

\section{CASO CLÍNICO}

S e trata de escolar femenina de 6 años de edad ingresada a hospitalización en el servicio de pediatría, con el diagnóstico de Neumonia Bilateral + Neurofibromatosis Tipo $1+$ LOE.

Antecedentes: padre con NF-1 sin complicaciones.

Presentando en la valoración saturación de oxígeno: 88\% (aire ambiente), pulso: 130xmin, talla: $106 \mathrm{~cm}$, peso: $21.600 \mathrm{~kg}$, circunferencia Braquial: $19 \mathrm{~cm}$, circunferencia Cefálica: $53 \mathrm{~cm} \mathrm{e}$ IMC: 19.28 .

\section{Valoración:}

Neurológico: Consciente, alerta, orientada en tiempo, espacio y persona; sueño conservado. Desviación observable de la columna (escoliosis), macrocefalia.

Respiratorio: Presencia de un trasqueostomo, ruidos agregados: crepitantes. Presencia de manchas color café con leche en tórax.

Cardiovascular: Ruidos cardiacos rítmicos y regulares, pulsos periféricos presentes y simétricos de amplitud conservada, llenado capilar de 2 segundos.
Gastrointestinal: Abdomen simétrico, superficie abdominal convexa, ruidos hidroaéreos presentes, patrón de eliminación: evacuación 1 vez al día. Presencia de manchas color café con leche.

Renal- Metabólico: No presenta dolor al palpar ni al percutir, patrón de eliminación: micciona 3 veces al día.

Posterior a la valoración de enfermería, se procedió a determinar los diagnósticos y establecer el plan de acción con base a las necesidades detectadas, utilizando el Proceso de Atención que es la metodología del trabajo enfermero estableciéndose:

Diagnósticos de enfermería. Estos se definieron con base a los criterios de la North American Nursing Diagnosis Association (NANDA) con el uso de:

Las Nursing Interventions Classification (NIC): Es una clasificación normalizada y completa de las intervenciones que realizan los profesionales de enfermería. Es útil para la planificación de los cuidados, la documentación clínica, la comunicación de cuidados en distintas situaciones, la integración de datos entre sistemas y situaciones, la investigación eficaz, la cuantificación de la productividad, la evaluación de la competencia, la retribución, la docencia y el diseño de programas $(9,10)$.

Los Nursing Outcomes (NOC) clasificación que describe los resultados que procedan de acciones de cuidados enfermeros y sean el resultado de los diagnósticos enfermeros identificados y finalmente, cómo y cuándo se realizará la medición de los resultados (10). El NOC plantea una estructura taxonómica en tres niveles: dominio, clases y resultado con sus indicadores.

Para el presente estudio de caso se determinaron dos diagnósticos de enfermería: Limpieza ineficaz de las vías aéreas $r / c$ retención de secreciones $m / p$ 
disnea y riesgo de infección r/c procedimiento invasivo.

1. Limpieza ineficaz de las vías aéreas $\mathbf{r} / c$ retención de secreciones $\mathrm{m} / \mathrm{p}$ disnea. Dominio 11, Clase 2, Código 00220 (NANDA).

NOC: Permeabilidad de las vías respiratorias.

NIC: Aspiración de las vías aéreas.

- Determinar la necesidad de la aspiración oral y/o traqueal.

- Auscultar los sonidos respiratorios antes y después de la aspiración.

- Informar al paciente y a la familia sobre el procedimiento a realizar de aspiración.

- Disponer precauciones universales: guantes, mascarilla.

- Utilizar equipo desechable estéril para cada procedimiento de aspiración traqueal.

- Seleccionar un catéter de aspiración adecuado (que sea la mitad del diámetro interior del tubo o vía aérea del paciente).

- Observar los niveles de SaO2 y ritmo cardiaco inmediatamente antes, durante y después de la succión.

- Ajustar la duración de la aspiración en la necesidad de extraer secreciones y en la respuesta del paciente a la aspiración.

- Anotar el tipo y cantidad de secreciones obtenidas.

Evaluación: se logra mejorar la permeabilidad de las vías aéreas.

NIC: Fisioterapia respiratoria.

- Utilizar y administrar nebulizaciones.

- Administrar broncodilatadores, si está indicado.

- Administrar agentes mucolíticos, si procede.

- Observar la tolerancia del paciente por medio de la SaO2, ritmo y frecuencia respiratoria, ritmo y frecuencia cardiacos y niveles de comodidad.

Evaluación: se evidencia que la paciente logra después de nebulizar mejorar el intercambio gaseoso.

2. Riesgo de infección $r / c$ procedimiento invasivo. Dominio 11 , Clase 1, Código 00004 (NANDA).

NOC: Integridad tisular: piel y membranas mucosas.

NIC: Observar los signos y síntomas de infección sistémica y localizada.

- Observar el grado de vulnerabilidad del paciente a las infecciones.

- Limitar el número de visitas, si procede.

- Analizar todas las visitas por si se padecen enfermedades transmisibles.

- Mantener las normas de asepsia para el paciente de riesgo.

- Inspeccionar la existencia de enrojecimiento, calor externo o drenaje en la piel y las membranas mucosas.

- Inspeccionar el estado de cualquier incisión / herida quirúrgica.

- Obtener muestras para realizar un cultivo, si es necesario. Facilitar el descanso.

- Observar si hay cambios en el nivel de vitalidad/malestar. Instruir al paciente y familiares acerca de los signos y síntomas de la infección y cuándo debe informar de ellos al cuidador. Enseñar al paciente y a la familia a evitar infecciones.

- La manipulación de la cánula, ya sea intubación, aseo de la cánula o cambio de ella debe realizarse con todas las normas de asepsia usando guantes estériles. Del mismo modo la aspiración traqueobronquial, toma de cultivo de las secreciones. 
- La cánula debe mantenerse en buena posición ya que al angularse puede erosionar la pared traqueal y provocar pequeñas hemorragias, úlceras, tejido de granulación, infecciones agregadas y estenosis posterior.

- Es prudente contar con 2 cánulas para ir alternándolas.

- Instilar algunas gotas de suero fisiológico al interior del lumen traqueal al momento de realizar la aspiración, induce accesos de tos que ayudan a eliminar las secreciones y además humidifica la mucosa del árbol respiratorio.

Evaluación: Se evitará la infección tanto del catéter venoso periférico, como del trasqueostomo, en su estadía hospitalaria.

Otras intervenciones de enfermería están dirigidas a mejorar el confort y promover el descanso de la escolar. Promover la interacción con otras personas. Instruir a padres y familiares a incluir actividades lúdicas de bajo impacto en su rutina diaria. Valorar diariamente el estado neurológico. Valorar el estado de la piel.

\section{DISCUSIÓN}

$\mathrm{L}$ a neurofibromatosis es una enfermedad poco frecuente, lo que la hace poco conocida dentro de los profesionales de la salud, dificultando así su diagnóstico y manejo. Esta patología con sintomatología muy variable no tiene cura, por lo que las intervenciones de enfermería están directamente basadas en proporcionar atención a las necesidades interferidas que presente la persona. De manera frecuente, sus necesidades están dirigidas hacia el sistema respiratorio y sistema motor con limitación muchas veces de la locomoción, brindar confort a la persona afectada en este caso una escolar y educación a los padres para que conozcan el manejo y las complicaciones que se pueden presentar.

Para el diagnóstico se consideró los criterios anteriormente mencionados de los cuales cumple con: 1 . Seis o más manchas "café con leche" mayores de 5 mm de diámetro en personas antes de la pubertad. 2. Pecas en las axilas y/o en inglés. 3. Una lesión ósea característica (como la escoliosis). 4. Pariente de primer grado afectado de NF1 (padre o hermano). Teniendo en cuenta los criterios anteriores, este paciente cuenta con cuatro de los criterios considerados para establecer un diagnóstico de NF-1. 

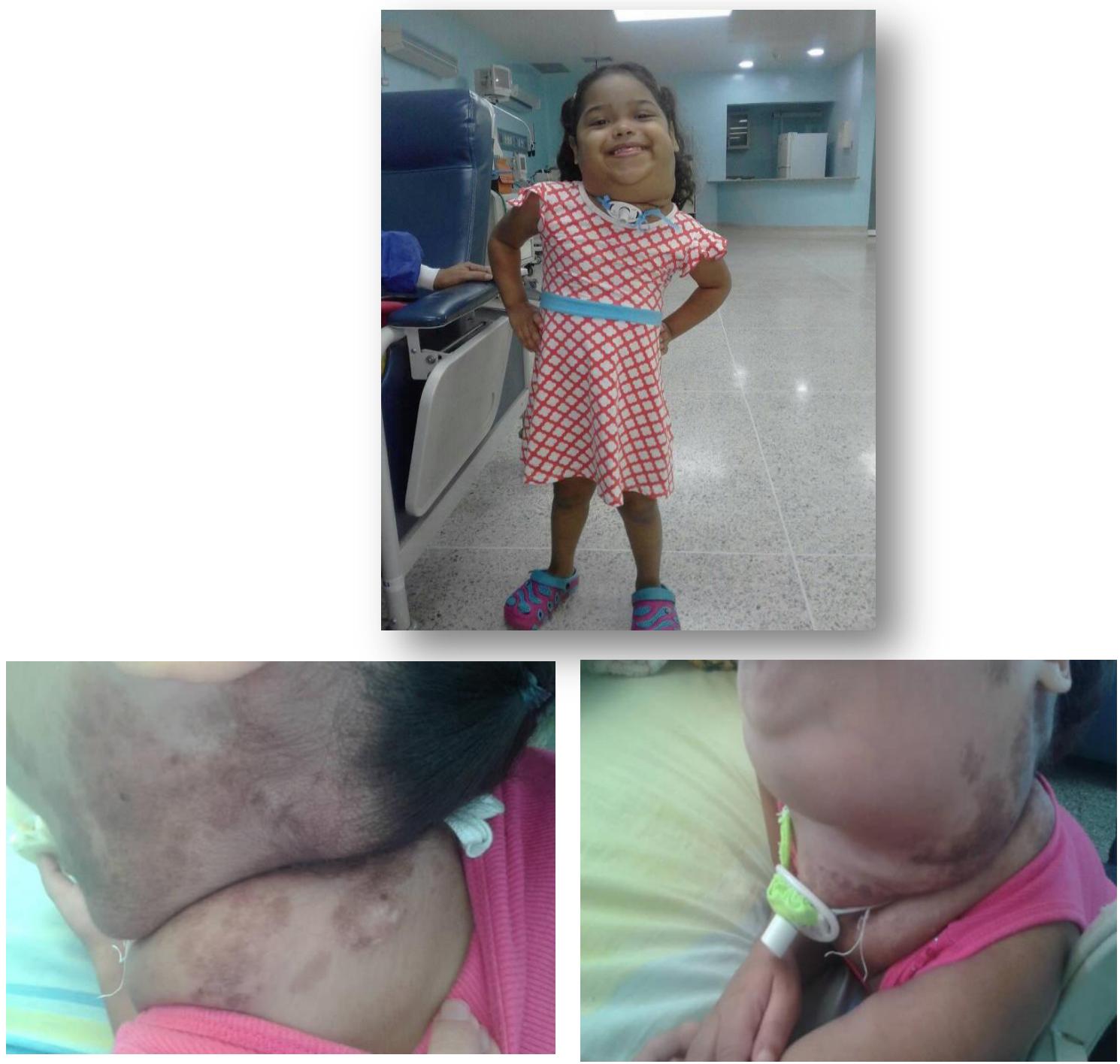

Imagen 1. Escolar donde se muestran los signos para establecer el diagnóstico y las complicaciones.

\section{CONCLUSIÓN}

$\mathrm{E}$ sta es una enfermedad que se caracteriza por alteraciones en la piel, neurofibromas, tumores en la vía óptica, cerebro, tracto digestivo, malformaciones cardiacas y alteraciones óseas. El diagnóstico se basa en criterios clínicos y estudios genéticos. No existe tratamiento específico para ella y su atención se basa en el tratamiento paliativo, las intervenciones de enfermería deben ser enfocadas hacia las necesidades interferidas que presenta y la educación a los padres. Es importante la utilización del proceso de enfermería, metodología que permitió realizar la valoración, establecer los diagnósticos, determinar un plan de acción y evaluar los resultados del cuidado una adecuada valoración de enfermería que sumada al conocimiento sobre el curso de la patología permitirá prevenir complicaciones futuras y educar a los padres en cuanto a la actuación y el apoyo como familia, estimular el intercambio con otros niños para conservar el sentido social, de pertenencia y valoración propia 
en los niños que padecen esta terrible enfermedad.

- Conflicto de intereses: Ninguno declarado por la autora.

- Fuente de financiamiento: Autofinanciado.

- Agradecimientos: Ninguno declarado por la autora.

\section{REFERENCIAS}

1. Enciclopedia Médica en Español: neurofibromatosis Madrid: Medlineplus; 2006 [Consultado 201815 noviembre]. Disponible en:

http://www.nlm.nih.gov/medlineplus/spanish/ ency/article/001289.ht

2. Boyd K, Korf B, Theos A.Neurofibromatosis type 1. J Am Acad Dermatol. [Internet] 2009 [Consultado 2018 el 15 noviembre]; 61(1): 116. doi;10.1016/j.jaad.2008.12.051

3. Quiroga E,Bermejo A, Fernández B. Neurofibromatosis: Neurofibroma plexiforme localizado en mama. A propósito de un caso. Rev. Argent. Dermatol. [Internet]. 2010 Mar [consultado $2018 \quad 10$ de diciembre] Disponible

en: http://www.scielo.org.ar/scielo.php?script =sci_arttext\&pid=S1851$300 \bar{X} 2010000100006 \&$ Ing $=$ es.

4. Ríos C,Mora G. Neuro-genetica. Neurofibromatosis tipo 1 -enfermedad de von Recklinghausen. Revista Médica de Costa Rica y Centroamérica LXXI (610) 249 - 252, 2014
5. García F. Estudio clínico ecográfico de la neurofibromatosis tipo 1 en la edad pediátrica. Universidad Autónoma de Madrid. Facultad de Medicina.2017

6. Fuentes Rodríguez N, Tápanes Domínguez A, Pérez La O P. Neurofibromatosis tipo I, enfermedad de von Recklinhausen. Rev Cubana Med Mil [Internet]. 2007 [consultado 201810 de octubre]; Disponible en: http://scielo.sld.cu/scielo.php?pid=S0138$65572007000400009 \&$ script=sci_arttext ISSN 1028-9933 350

7. U.S. Department of Health \& Human Services Neurofibromatosis tipo 1. En: Enfermedades raras [Internet [consultado 201810 de octubre] Disponible en: http://www.intramed.net/contenidover.asp?co ntenidolD=48296

8. Rodríguez L, Carneiro O, Batista P, GoloniBertollo E, Souza-Costa D, Eliam L et al. Das neurofibromatoses: parte 1 diagnóstico e diagnóstico diferencial. Arq. Neuro-Psiquiatr. [Internet]. 2014 [consultado 2018 el 10 de octubre].Doi_org/10.1590/0004282X20130241.

9. Bavaresco T, Lucena A. Intervenciones de la Clasificación de Enfermería NIC validadas para pacientes en riesgo de úlcera por presión. Rev. Latino-Am. Enfermagem [Internet]. 2012 Dec [citado 2019 febrero 04]; 20(6): 1109-1116. http://dx.doi.org/10.1590/S010411692012000600013

10. Johnson M, Maas M, Moorhead S. lowa Outcomes Project. Nursing Outcomes Classification (NOC). 2da Ed: Philadelphia: Ed. Mosby; 2000 